九州大学学術情報リポジトリ

Kyushu University Institutional Repository

\title{
SUCCESSIVE COMPARISONS BETWEEN ORDERED NORMAL MEANS BASED ON CLOSED TESTING PROCEDURE
}

Imada, Tsunehisa

Liberal Arts Education Center, Kumamoto Campus, Tokai University

https://doi.org/10.5109/1906488

出版情報: Bulletin of informatics and cybernetics. 47, pp.25-36，2015-12. Research Association of Statistical Sciences

バージョン：

権利関係 : 


\title{
SUCCESSIVE COMPARISONS BETWEEN ORDERED NORMAL MEANS BASED ON CLOSED TESTING PROCEDURE
}

\author{
By \\ Tsunehisa IMADA*
}

\begin{abstract}
In this study we discuss multiple tests for checking differences among a sequence of normal means with ordered restriction. Specifically, we propose a stepwise multiple comparison procedure based on closed testing procedure using the statistics used by Williams (1971). We give some numerical examples regarding critical values and power of the test intended to compare our procedure and other procedures.
\end{abstract}

Key Words and Phrases: Multivariate $t$-distribution, Power of the test, Stepwise test.

\section{Introduction}

There are independent normal random variables $X_{1}, X_{2}, \ldots, X_{K}$. Assume

$$
X_{k} \sim N\left(\mu_{k}, \sigma^{2}\right)
$$

for $k=1,2, \ldots, K$. Assuming

$$
\mu_{1} \leq \mu_{2} \leq \cdots \leq \mu_{K}
$$

we consider multiple tests to find differences among $\mu_{1}, \mu_{2}, \cdots, \mu_{K}$. Lee and Spurrier (1995) set up the null hypothesis and its alternative hypothesis as

$$
H_{k, k+1}: \mu_{k}=\mu_{k+1} \text { vs. } H_{k, k+1}^{A}: \mu_{k}<\mu_{k+1}
$$

for $k=1,2, \ldots, K-1$ and proposed a single step multiple comparison procedure for $H_{1,2}$, $H_{2,3}, \ldots, H_{K-1, K}$. Shiraishi (2014) developed Lee and Spurrier (1995)'s procedure using closed testing procedure intended to obtain a more powerful procedure. Specifically, Shiraishi (2014) constructed the closed set of hypotheses $F$ based on $H_{1,2}, H_{2,3}, \ldots, H_{K-1, K}$ and proposed a stepwise multiple comparison procedure for $F$ based on closed testing procedure. Furthermore, Douke et al. (2006) proposed a sequential rejective step down procedure for $H_{1,2}, H_{2,3}, \ldots, H_{K-1, K}$ and Imada (2015) proposed a step up procedure for them.

On the other hand, Hayter (1990) set up the null hypothesis and its alternative hypothesis as

$$
H_{k, l}: \mu_{k}=\mu_{l} \text { vs. } H_{k, l}^{A}: \mu_{k}<\mu_{l}
$$

* Liberal Arts Education Center, Kumamoto Campus, Tokai University 9-1-1, Toroku Higashi-ku Kumamoto, Japan 862-8652 Japan. tel +81-96-386-2731 timada@ktmail.tokai-u.jp 
for $1 \leq k<l \leq K$ and proposed a single step multiple comparison procedure for all $H_{k, l}$ s. Furthermore, Shiraishi (2014) developed Hayter (1990)'s procedure using closed testing procedure.

However, the assumption (1) was not adopted in constructing the procedures described above. The isotonic regression estimators of $\mu_{1}, \mu_{2}, \ldots, \mu_{K}$ are the maximum likelihood estimators under (1). Nashimoto and Wright (2005) proposed two types of multiple comparison procedures for all $H_{k, l} \mathrm{~s}(1 \leq k<l \leq K)$ using the isotonic regression estimators. Furthermore, they modified the procedures to avoid the contradiction that $H_{k, l}$ is retained and $H_{k^{\prime}, l^{\prime}}$ is rejected for $1 \leq k \leq k^{\prime}<l^{\prime} \leq l \leq K$.

On the other hand, Williams (1971) proposed a step down multiple comparison procedure for finding minimum $k$ satisfying $\mu_{k}<\mu_{k+1}$ based on the statistics using the isotonic regression estimators.

In this study we focus on the simultaneous test for $H_{1,2}, H_{2,3}, \ldots, H_{K-1, K}$ and propose another type of stepwise multiple comparison procedure based on closed testing procedure using Williams (1971)'s statistics.

In Section 2, we discuss Lee and Spurrier (1995)'s single step multiple comparison procedure. Specifically, we discuss how to determine the critical value for a specified significance level according to Lee and Spurrier (1995). Furthermore, we formulate the power of the test. In Section 3, we discuss Shiraishi (2014)'s stepwise multiple comparison based on closed testing procedure. In Section 4, we discuss a stepwise multiple comparison based on closed testing procedure using Williams (1971)'s statistics. In Section 5, we give some numerical examples regarding critical values and power of the test intended to compare the procedures. In Section 6, we give some concluding remark.

\section{Lee and Spurrier (1995)'s single step procedure}

\subsection{Determination of the critical value for a specified significance level}

Let $x_{k 1}, x_{k 2}, \ldots, x_{k n_{k}}$ be a sample from $N\left(\mu_{k}, \sigma^{2}\right)$ for $k=1,2, \ldots, K$. Lee and Spurrier (1995) used the statistic

$$
t_{k, k+1}=\frac{\bar{x}_{k+1}-\bar{x}_{k}}{\sqrt{\frac{1}{n_{k+1}}+\frac{1}{n_{k}}} s}
$$

for testing $H_{k, k+1}$. Here

$$
\bar{x}_{k}=\frac{1}{n_{k}} \sum_{i=1}^{n_{k}} x_{k i}, \quad s=\sqrt{\frac{1}{\phi} \sum_{k=1}^{K} \sum_{i=1}^{n_{k}}\left(x_{k i}-\bar{x}_{k}\right)^{2}}
$$

where $\phi=\sum_{k=1}^{K} n_{k}-K$. If $t_{k, k+1}>c$ for a specified critical value $c, H_{k, k+1}$ is rejected. Otherwise, it is retained. The critical value $c$ is determined so that

$$
P\left(\max _{1 \leq k \leq K-1} t_{k, k+1}>c\right)=\alpha
$$

for a specified significance level $\alpha$ under the assumption that $H_{1,2}, H_{2,3}, \ldots, H_{K-1, K}$ are true. The left hand of (2) is the probability that at least one hypothesis among $H_{1,2}, H_{2,3}, \ldots, H_{K-1, K}$ is rejected. (2) is equivalent to

$$
P\left(t_{1,2} \leq c, t_{2,3} \leq c, \ldots, t_{K-1, K} \leq c\right)=1-\alpha .
$$


We discuss how to calculate $P\left(t_{1,2} \leq c, t_{2,3} \leq c, \ldots, t_{K-1, K} \leq c\right)$ when $H_{1,2}, H_{2,3}, \ldots$, $H_{K-1, K}$ are true. $\left(t_{1,2}, t_{2,3}, \ldots, t_{K-1, K}\right)$ is distributed according to the multivariate $t$-distribution with covariance matrix

$$
\boldsymbol{\Lambda}=\left(\begin{array}{ccccccc}
1 & \rho_{1,2,3} & 0 & 0 & \cdots & 0 & 0 \\
\rho_{1,2,3} & 1 & \rho_{2,3,4} & 0 & \cdots & 0 & 0 \\
0 & \rho_{2,3,4} & 1 & \rho_{3,4,5} & \cdots & 0 & 0 \\
\vdots & \vdots & \vdots & \vdots & \vdots & \vdots & \vdots \\
0 & 0 & \cdots & 0 & \cdots & 1 & \rho_{K-2, K-1, K} \\
0 & 0 & \cdots & 0 & \cdots & \rho_{K-2, K-1, K} & 1
\end{array}\right)
$$

and $\phi$ degrees of freedom. Here

$$
\rho_{k, k+1, k+2}=-\sqrt{\frac{n_{k} n_{k+2}}{\left(n_{k}+n_{k+1}\right)\left(n_{k+1}+n_{k+2}\right)}} .
$$

Its provability density function is given by

$$
f\left(t_{1,2}, t_{2,3}, \ldots, t_{K-1, K} ; \boldsymbol{\Lambda}, \phi\right)=\frac{\Gamma((K-1+\phi) / 2))}{(\phi \pi)^{(K-1) / 2} \Gamma(\phi / 2)|\boldsymbol{\Lambda}|^{1 / 2}}\left(1+\frac{1}{\phi} \boldsymbol{t}^{\prime} \boldsymbol{\Lambda}^{-1} \boldsymbol{t}\right)^{-(K-1+\phi) / 2}
$$

where $\boldsymbol{t}=\left(t_{1,2}, t_{2,3}, \ldots, t_{K-1, K}\right)^{\prime}$. Then

$$
\begin{gathered}
P\left(t_{1,2} \leq c, t_{2,3} \leq c, \ldots, t_{K-1, K} \leq c\right) \\
=\int_{-\infty}^{c} \int_{-\infty}^{c} \cdots \int_{-\infty}^{c} f\left(t_{1,2}, t_{2,3}, \ldots, t_{K-1, K} ; \boldsymbol{\Lambda}, \phi\right) d t_{1,2} d t_{2,3} \cdots d t_{K-1, K} .
\end{gathered}
$$

\subsection{Power of the test}

Next, we consider the power of the test. Assume

$$
1 \leq i_{1}<i_{2}<\cdots<i_{k} \leq K-1
$$

and

$$
\mu_{i_{j}}<\mu_{i_{j}+1}(j=1,2, \ldots, k), \quad \mu_{l}=\mu_{l+1} \quad\left(l \neq i_{1}, i_{2}, \ldots, i_{k}\right) .
$$

We focus on the all pairs power defined by Ramsey (1978). It is the probability that $H_{i_{1}, i_{1}+1}, H_{i_{2}, i_{2}+1}, \ldots, H_{i_{k}, i_{k}+1}$ are rejected. The power is given by

$$
P\left(t_{i_{1}, i_{1}+1}>c, t_{i_{2}, i_{2}+1}>c, \ldots, t_{i_{k}, i_{k}+1}>c\right) .
$$

To calculate (4) we should specify the difference $\mu_{i_{j}+1}-\mu_{i_{j}}$ for $j=1,2, \ldots, k$ in (3). Specifically, let

$$
\mu_{i_{j}+1}-\mu_{i_{j}}=\delta_{i_{j}}(>0) \quad(j=1,2, \ldots, k), \quad \mu_{l}=\mu_{l+1} \quad\left(l \neq i_{1}, i_{2}, \ldots, i_{k}\right) .
$$

We discuss how to calculate (4) under (5). Letting

$$
t_{0, i_{j}, i_{j}+1}=\frac{\bar{x}_{i_{j}+1}-\bar{x}_{i_{j}}}{\sqrt{\frac{1}{n_{i_{j}+1}}+\frac{1}{n_{i_{j}}}} \sigma}(j=1,2, \ldots, k), \quad s_{0}=\frac{s}{\sigma},
$$


we obtain

$$
t_{i_{j}, i_{j}+1}=\frac{t_{0, i_{j}, i_{j}+1}}{s_{0}} \quad(j=1,2, \ldots, k)
$$

and

$$
\begin{gathered}
P\left(t_{i_{1}, i_{1}+1}>c, t_{i_{2}, i_{2}+1}>c, \ldots, t_{i_{k}, i_{k}+1}>c\right) \\
=P\left(t_{0, i_{1}, i_{1}+1}>c s_{0}, t_{0, i_{2}, i_{2}+1}>c s_{0}, \ldots, t_{0, i_{k}, i_{k}+1}>c s_{0}\right) .
\end{gathered}
$$

$\left(t_{0, i_{1}, i_{1}+1}, t_{0, i_{2}, i_{2}+1}, \ldots, t_{0, i_{k}, i_{k}+1}\right)^{\prime}$ is distributed according to the multivariate normal $N_{k}\left(\boldsymbol{\delta}, \boldsymbol{\Lambda}_{1}\right)$ where

$$
\boldsymbol{\delta}=\left(\frac{\delta_{i_{1}}}{\sqrt{\frac{1}{n_{i_{1}}+1}+\frac{1}{n_{i_{1}}}} \sigma}, \frac{\delta_{i_{2}}}{\sqrt{\frac{1}{n_{i_{2}}+1}+\frac{1}{n_{i_{2}}}} \sigma}, \ldots, \frac{\delta_{i_{k}}}{\sqrt{\frac{1}{n_{i_{k}}+1}+\frac{1}{n_{i_{k}}}} \sigma}\right)^{\prime}
$$

and $\boldsymbol{\Lambda}_{1}$ is the submatrix of $\boldsymbol{\Lambda}$. When the value of $s_{0}$ is given,

$$
\begin{gathered}
P\left(t_{0, i_{1}, i_{1}+1}>c s_{0}, t_{0, i_{2}, i_{2}+1}>c s_{0}, \ldots, t_{0, i_{k}, i_{k}+1}>c s_{0} \mid s_{0}\right) \\
=\int_{c s_{0}}^{\infty} \int_{c s_{0}}^{\infty} \cdots \int_{c s_{0}}^{\infty} f_{1}\left(t_{0, i_{1}, i_{1}+1}, t_{0, i_{2}, i_{2}+1}, \ldots, t_{0, i_{k}, i_{k}+1} ; \boldsymbol{\delta}, \boldsymbol{\Lambda}_{1}\right) \\
\times d t_{0, i_{1}, i_{1}+1} d t_{0, i_{2}, i_{2}+1} \cdots d t_{0, i_{k}, i_{k}+1}
\end{gathered}
$$

where $f_{1}\left(t_{0, i_{1}, i_{1}+1}, t_{0, i_{2}, i_{2}+1}, \ldots, t_{0, i_{k}, i_{k}+1} ; \boldsymbol{\delta}, \boldsymbol{\Lambda}_{1}\right)$ is the probability density function of $N_{k}\left(\boldsymbol{\delta}, \boldsymbol{\Lambda}_{1}\right)$. On the other hand, since $\phi s_{0}^{2}$ is distributed according to $\chi^{2}$-distribution with $\phi$ degrees of freedom, the probability density function of $s_{0}$ is

$$
g\left(s_{0}\right)=\frac{\phi^{\phi / 2}}{2^{(\phi-2) / 2} \Gamma[\phi / 2]} s_{0}^{\phi-1} \exp \left[-\frac{\phi s_{0}^{2}}{2}\right] .
$$

The power is given by

$$
\begin{gathered}
P\left(t_{i_{1}, i_{1}+1}>c, t_{i_{2}, i_{2}+1}>c, \ldots, t_{i_{k}, i_{k}+1}>c\right) \\
=\int_{0}^{\infty} g\left(s_{0}\right)\left\{\int_{c s_{0}}^{\infty} \int_{c s_{0}}^{\infty} \cdots \int_{c s_{0}}^{\infty} f_{1}\left(t_{0, i_{1}, i_{1}+1}, t_{0, i_{2}, i_{2}+1}, \ldots, t_{0, i_{k}, i_{k}+1} ; \boldsymbol{\delta}, \boldsymbol{\Lambda}_{1}\right)\right. \\
\left.\times d t_{0, i_{1}, i_{1}+1} d t_{0, i_{2}, i_{2}+1} \cdots d t_{0, i_{k}, i_{k}+1}\right\} d s_{0} .
\end{gathered}
$$

Since $\boldsymbol{\delta}$ depends on the unknown $\sigma$, we should specify the value of $\sigma$ to calculate the power.

\section{Shiraishi (2014)'s stepwise procedure based on closed testing procedure}

Let $F$ be the family of hypotheses consisting of $H_{1,2}, H_{2,3}, \ldots, H_{K-1, K}$ and all sorts of intersections of plural hypotheses chosen from $H_{1,2}, H_{2,3}, \ldots, H_{K-1, K}$. $F$ is closed. Specifically, for two hypotheses chosen from $F$ arbitrarily, their intersection is also included in $F$. Shiraishi (2014) proposed a stepwise multiple comparison procedure for $F$ based on closed testing procedure. 
Let $I$ be a subset of $\{1,2, \ldots, K-1\}$ consisting of successive plural integers. $\sharp(I)$ denotes the number of elements of $I$. Therefore, if $I=\{i, i+1, \ldots, j\}, \sharp(I)=j-i+1$. Defining the hypothesis $H_{I}$ as

$$
H_{I}: \mu_{i}=\mu_{i+1}=\cdots=\mu_{j}
$$

we obtain

$$
H_{I}=H_{i, i+1} \cap H_{i+1, i+2} \cap \cdots \cap H_{j-1, j} \in F .
$$

Occasionally, $H_{I}$ is denoted by $H_{i, j}$. Each hypothesis in $F$ is equal to $H_{I}$ or $H_{I_{1}} \cap H_{I_{2}} \cap$ $\cdots \cap H_{I_{k}}$ where $I_{1}, I_{2}, \ldots, I_{k}$ are disjoint. Intended to construct a stepwise procedure for $F$ based on closed testing procedure, we specify a way to test each hypothesis in $F$. We use the statistic $t_{I}=\max \left\{t_{i, i+1}, t_{i+1, i+2}, \ldots, t_{j-1, j}\right\}$ for testing $H_{I}: \mu_{i}=\mu_{i+1}=\cdots=$ $\mu_{j}$ and determine the critical value $c_{I}$ so that $P\left(t_{I}>c_{I}\right)=\alpha$ under the assumption that $H_{I}$ is true. If $t_{I}>c_{I}, H_{I}$ is rejected. Otherwise, it is retained.

Next, we discuss how to test $H_{I_{1}} \cap H_{I_{2}} \cap \cdots \cap H_{I_{k}}$ where $I_{1}, I_{2}, \ldots, I_{k}$ are disjoint. Let $M=\sharp\left(I_{1}\right)+\sharp\left(I_{2}\right)+\cdots+\sharp\left(I_{k}\right)$. For $l=1,2, \ldots, k$ we determine $c_{I_{l}, M}$ so that

$$
P\left(t_{I_{l}}>c_{I_{l}, M}\right)=1-(1-\alpha)^{\sharp\left(I_{l}\right) / M}
$$

under the assumption that $H_{I_{l}}$ is true. Specifically, intended to test $H_{I_{1}} \cap H_{I_{2}} \cap \cdots \cap H_{I_{k}}$ we set up the critical value $c_{I_{l}, M}$ for testing $H_{I_{l}}$ for $l=1,2, \ldots, k$. If $t_{I_{l}}>c_{I_{l}, M}$ for at least one $l, H_{I_{1}} \cap H_{I_{2}} \cap \cdots \cap H_{I_{k}}$ is rejected. Otherwise, it is retained. Shiraishi (2014) indicated the probability that $H_{I_{1}} \cap H_{I_{2}} \cap \cdots \cap H_{I_{k}}$ is rejected when it is true is not greater than $\alpha$. Therefore, we specified the way to test each hypothesis in $F$ satisfying the specified significance level $\alpha$. We test the hypotheses in $F$ hierarchically. Specifically, if a hypothesis and all hypotheses deriving it are rejected, we reject the hypothesis. Otherwise we retain it. By the principle of closed testing procedure maximum type I FWE (familywise error rate) of this stepwise procedure is not greater than $\alpha$. It is indicated by Nagata and Yoshida (1997).

Example.

Letting $K=4$,

$$
F=\left\{H_{1,4}, H_{1,3}, H_{2,4}, H_{1,2} \cap H_{3,4}, H_{1,2}, H_{2,3}, H_{3,4}\right\} .
$$

We test the hypotheses in $F$ as follows.

\section{Step 1.}

We test $H_{1,4}$.

Case 1. If $H_{1,4}$ is retained, we retain all other hypotheses in $F$ and stop the test.

Case 2. If $H_{1,4}$ is rejected, go to Step 2.

\section{Step 2.}

We test $H_{1,3}, H_{2,4}, H_{1,2} \cap H_{3,4}$.

If $H_{1,3}$ is retained, we retain $H_{1,2}, H_{2,3}$.

If $H_{2,4}$ is retained, we retain $H_{2,3}, H_{3,4}$.

If $H_{1,2} \cap H_{3,4}$ is retained, we retain $H_{1,2}, H_{3,4}$.

\section{Step 3.}

We test the hypotheses which are not retained at Step 2 among $H_{1,2}, H_{2,3}, H_{3,4}$.

The power under (5) is the probability that $H_{i_{1}, i_{1}+1}, H_{i_{2}, i_{2}+1}, \ldots, H_{i_{k}, i_{k}+1}$ are rejected at the final step in the stepwise test. Since it is difficult to formulate the probability, we calculate it by Monte Carlo simulation. 


\section{Stepwise procedure based on closed testing procedure using Williams (1971)'s statistic}

In Section 3 we discussed the stepwise procedure for $F$ based on closed testing procedure proposed by Shiraishi (2014). In this section we propose a stepwise procedure for $F$ using Williams (1971)'s statistic. First, we consider testing $H_{I}: \mu_{i}=\mu_{i+1}=\cdots=$ $\mu_{j}$. Let

$$
y_{l}=\frac{n_{l} \bar{x}_{l}+n_{l+1} \bar{x}_{l+1}+\cdots+n_{j} \bar{x}_{j}}{n_{(l, j)}}(l=i+1, i+2, \ldots, j)
$$

where $n_{(l, j)}=n_{l}+n_{l+1}+\cdots+n_{j} . M_{I}=\max \left\{y_{i+1}, y_{i+2}, \ldots, y_{j}\right\}$ is the isotonic regression estimator of $\mu_{j}$ under $\mu_{i} \leq \mu_{i+1} \leq \cdots \leq \mu_{j}$. We use

$$
t_{I}^{*}=\frac{M_{I}-\bar{x}_{i}}{\sqrt{\frac{1}{n_{j}}+\frac{1}{n_{i}}} s}
$$

for testing $H_{I}$. If $t_{I}^{*}>c_{I}^{*}, H_{I}$ is rejected. Otherwise, it is retained. We determine $c_{I}^{*}$ so that

$$
P\left(t_{I}^{*}>c_{I}^{*}\right)=\alpha
$$

under the assumption that $H_{I}$ is true. (6) is equivalent to

$$
P\left(t_{I}^{*} \leq c_{I}^{*}\right)=1-\alpha .
$$

To determine $c_{I}^{*}$ we consider how to calculate $P\left(t_{I}^{*} \leq c_{I}^{*}\right)$. Letting

$$
z_{l}=\frac{y_{l}-\bar{x}_{i}}{\sqrt{\frac{1}{n_{j}}+\frac{1}{n_{i}} s}}
$$

for $l=i+1, i+2, \ldots, j$, we obtain

$$
P\left(t_{I}^{*} \leq c_{I}^{*}\right)=P\left(z_{i+1} \leq c_{I}^{*}, z_{i+2} \leq c_{I}^{*}, \ldots, z_{j} \leq c_{I}^{*}\right) .
$$

$\left(z_{i+1}, z_{i+2}, \ldots, z_{j}\right)^{\prime}$ is distributed according to the multivariate $t$-distribution with covariance matrix

$$
\boldsymbol{\Lambda}_{I}=\frac{1}{\frac{1}{n_{j}}+\frac{1}{n_{i}}}\left(\begin{array}{ccccc}
\frac{1}{n_{i}}+\frac{1}{n_{(i+1, j)}} & \frac{1}{n_{i}}+\frac{1}{n_{(i+1, j)}} & \frac{1}{n_{i}}+\frac{1}{n_{(i+1, j)}} & \cdots & \frac{1}{n_{i}}+\frac{1}{n_{(i+1, j)}} \\
\frac{1}{n_{i}}+\frac{1}{n_{(i+1, j)}} & \frac{1}{n_{i}}+\frac{1}{n_{(i+2, j)}} & \frac{1}{n_{i}}+\frac{1}{n_{(i+2, j)}} & \cdots & \frac{1}{n_{i}}+\frac{1}{n_{(i+2, j)}} \\
\frac{1}{n_{i}}+\frac{1}{n_{(i+1, j)}} & \frac{1}{n_{i}}+\frac{1}{n_{(i+2, j)}} & \frac{1}{n_{i}}+\frac{1}{n_{(i+3, j)}} & \cdots & \frac{1}{n_{i}}+\frac{1}{n_{(i+3, j)}} \\
\vdots & \vdots & \vdots & \ddots & \vdots \\
\frac{1}{n_{i}}+\frac{1}{n_{(i+1, j)}} & \frac{1}{n_{i}}+\frac{1}{n_{(i+2, j)}} & \frac{1}{n_{i}}+\frac{1}{n_{(i+3, j)}} & \cdots & \frac{1}{n_{(j-1, j)}}+\frac{1}{n_{i}} \\
\frac{1}{n_{i}}+\frac{1}{n_{(i+1, j)}} & \frac{1}{n_{i}}+\frac{1}{n_{(i+2, j)}} & \frac{1}{n_{i}}+\frac{1}{n_{(i+3, j)}} & \cdots & \frac{1}{n_{i}}+\frac{1}{n_{j}}
\end{array}\right)
$$

and $\phi$ degrees of freedom. Letting $f\left(z_{i+1}, z_{i+2}, \ldots, z_{j} ; \boldsymbol{\Lambda}_{I}, \phi\right)$ be its provability density function, we obtain

$$
\begin{aligned}
P\left(t_{I}^{*} \leq c_{I}^{*}\right) & =P\left(z_{i+1} \leq c_{I}^{*}, z_{i+2} \leq c_{I}^{*}, \ldots, z_{j} \leq c_{I}^{*}\right) \\
& =\int_{-\infty}^{c_{I}^{*}} \int_{-\infty}^{c_{I}^{*}} \cdots \int_{-\infty}^{c_{I}^{*}} f\left(z_{i+1}, z_{i+2}, \ldots, z_{j} ; \boldsymbol{\Lambda}_{I}, \phi\right) d z_{i+1} d z_{i+2} \cdots d z_{j}
\end{aligned}
$$


Next, we discuss how to test $H_{I_{1}} \cap H_{I_{2}} \cap \cdots \cap H_{I_{k}}$ where $I_{1}, I_{2}, \ldots, I_{k}$ are disjoint. Let $M=\sharp\left(I_{1}\right)+\sharp\left(I_{2}\right)+\cdots+\sharp\left(I_{k}\right)$. For $l=1,2, \ldots, k$ we determine $c_{I_{l}, M}^{*}$ so that

$$
P\left(t_{I_{l}}^{*}>c_{I_{l}, M}^{*}\right)=1-(1-\alpha)^{\sharp\left(I_{l}\right) / M}
$$

under the assumption that $H_{I_{l}}$ is true. If $t_{I_{l}}^{*}>c_{I_{l}, M}^{*}$ for at least one $l, H_{I_{1}} \cap H_{I_{2}} \cap \cdots \cap H_{I_{k}}$ is rejected. Otherwise, it is retained. Therefore, the probability that $H_{I_{1}} \cap H_{I_{2}} \cap \cdots \cap H_{I_{k}}$ is rejected is

$$
1-P\left(t_{I_{1}}^{*} \leq c_{I_{1}, M}^{*}, t_{I_{2}}^{*} \leq c_{I_{2}, M}^{*}, \ldots, t_{I_{k}}^{*} \leq c_{I_{k}, M}^{*}\right) .
$$

When $H_{I_{1}}, H_{I_{2}}, \cdots, H_{I_{k}}$ are true,

$$
1-P\left(t_{I_{1}}^{*} \leq c_{I_{1}, M}^{*}, t_{I_{2}}^{*} \leq c_{I_{2}, M}^{*}, \ldots, t_{I_{k}}^{*} \leq c_{I_{k}, M}^{*}\right) \leq \alpha,
$$

because

$$
P\left(t_{I_{1}}^{*} \leq c_{I_{1}, M}^{*}, t_{I_{2}}^{*} \leq c_{I_{2}, M}^{*}, \ldots, t_{I_{k}}^{*} \leq c_{I_{k}, M}^{*}\right) \geq \prod_{l=1}^{k} P\left(t_{I_{l}}^{*} \leq c_{I_{l}, M}^{*}\right)
$$

and

$$
\prod_{l=1}^{k} P\left(t_{I_{l}}^{*} \leq c_{I_{l}, M}^{*}\right)=1-\alpha .
$$

The proof of (7) is given in Appendix. Since we specified the way to test each hypothesis in $F$ satisfying the specified significance level $\alpha$, the stepwise procedure based on closed testing procedure is constructed.

It is also difficult to formulate the power of the test under (5). The power is calculated by Monte Carlo simulation.

\section{Simulation results}

We discussed three types of multiple comparison procedures intended to find differences among a sequence of normal means with ordered restriction. First, we discussed Lee and Spurrier (1995)'s single step procedure. Next, we discussed Shiraishi (2014)'s stepwise procedure developing Lee and Spurrier (1995)'s procedure based on closed testing procedure. Furthermore, we proposed the stepwise procedure based on closed testing procedure using Williams (1971)'s statistics. In this section we give some simulation results intended to compare five procedures including Douke et al. (2006)'s sequential rejective step down procedure and Imada (2015)'s step up procedure. SS, CT, CT-W, $\mathrm{SD}$ and $\mathrm{SU}$ denote these procedures, respectively.

Let $K=3,4,5$ and $\alpha=0.05$. We set up a balanced sample size $n$ for each population. Specifically, let $n=15,30$. Table 1 gives critical values of SS.

Table 1 : Critical values of SS

\begin{tabular}{c|ccc}
\hline$K$ & 3 & 4 & 5 \\
\hline$n=15$ & 2.018 & 2.179 & 2.286 \\
$n=30$ & 1.988 & 2.152 & 2.261 \\
\hline
\end{tabular}


Tables 2,3,4 give critical values of $\mathrm{CT}$ for $K=3,4,5$. When we test $H_{1,3} \cap$ $H_{4,5}, H_{1,2} \cap H_{3,5}$ by Table 4 , we use the critical values $2.211,2.188$ for testing $H_{1,3}$ and $H_{3,5}$ and use 2.087, 2.066 for testing $H_{4,5}$ and $H_{1,2}$ for $n=15,30$, respectively. Tables $5,6,7$ give critical values of CT-W for $K=3,4,5$. When we test $H_{1,3} \cap H_{4,5}, H_{1,2} \cap H_{3,5}$ in Table 7 , we use the critical values $1.970,1.953$ for testing $H_{1,3}$ and $H_{3,5}$ and use 2.087, 2.066 for testing $H_{4,5}$ and $H_{1,2}$ for $n=15,30$, respectively.

Table 2: Critical values of $\mathrm{CT}(K=3)$

\begin{tabular}{c|cc}
\hline & $n=15$ & $n=30$ \\
\hline$H_{1,3}$ & 2.018 & 1.988 \\
$H_{1,2}, H_{2,3}$ & 1.682 & 1.663 \\
\hline
\end{tabular}

Table 3 : Critical values of CT $(K=4)$

\begin{tabular}{c|cc}
\hline & $n=15$ & $n=30$ \\
\hline$H_{1,4}$ & 2.179 & 2.152 \\
$H_{1,3}, H_{2,4}$ & 2.004 & 1.981 \\
$H_{1,2} \cap H_{3,4}$ & 1.998 & 1.976 \\
$H_{1,2}, H_{2,3}, H_{3,4}$ & 1.673 & 1.659 \\
\hline
\end{tabular}

Table 4 : Critical values of $\mathrm{CT}(K=5)$

\begin{tabular}{c|cc}
\hline & $n=15$ & $n=30$ \\
\hline$H_{1,5}$ & 2.286 & 2.261 \\
$H_{1,4}, H_{2,5}$ & 2.168 & 2.146 \\
$H_{1,3} \cap H_{4,5}, H_{1,2} \cap H_{3,5}$ & $2.211,2.087$ & $2.188,2.066$ \\
$H_{1,3}, H_{2,4}, H_{3,5}$ & 1.995 & 1.977 \\
$H_{1,2} \cap H_{3,4}, H_{1,2} \cap H_{4,5}, H_{2,3} \cap H_{4,5}$ & 1.989 & 1.971 \\
$H_{1,2}, H_{2,3}, H_{3,4}, H_{4,5}$ & 1.667 & 1.656 \\
\hline
\end{tabular}

Table 5 : Critical values of CT-W $(K=3)$

\begin{tabular}{c|cc}
\hline & $n=15$ & $n=30$ \\
\hline$H_{1,3}$ & 1.758 & 1.737 \\
$H_{1,2}, H_{2,3}$ & 1.682 & 1.663 \\
\hline
\end{tabular}

Table 6 : Critical values of CT-W $(K=4)$

\begin{tabular}{c|cc}
\hline & $n=15$ & $n=30$ \\
\hline$H_{1,4}$ & 1.772 & 1.755 \\
$H_{1,3}, H_{2,4}$ & 1.748 & 1.732 \\
$H_{1,2} \cap H_{3,4}$ & 1.998 & 1.976 \\
$H_{1,2}, H_{2,3}, H_{3,4}$ & 1.673 & 1.659 \\
\hline
\end{tabular}

Table 7 : Critical values of CT-W $(K=5)$

\begin{tabular}{c|cc}
\hline & $n=15$ & $n=30$ \\
\hline$H_{1,5}$ & 1.777 & 1.763 \\
$H_{1,4}, H_{2,5}$ & 1.766 & 1.752 \\
$H_{1,3} \cap H_{4,5}, H_{1,2} \cap H_{3,5}$ & $1.970,2.087$ & $1.953,2.066$ \\
$H_{1,3}, H_{2,4}, H_{3,5}$ & 1.742 & 1.729 \\
$H_{1,2} \cap H_{3,4}, H_{1,2} \cap H_{4,5}, H_{2,3} \cap H_{4,5}$ & 1.989 & 1.971 \\
$H_{1,2}, H_{2,3}, H_{3,4}, H_{4,5}$ & 1.667 & 1.656 \\
\hline
\end{tabular}


Next, we give numerical examples of power of the test. Let $\sigma=1$. We set up two types of arrangements of means $\left(\mu_{1}, \mu_{2}, \mu_{3}\right)=(0,1,2),(0,1,1)$ for $K=3$. We set up three types of arrangements of means $\left(\mu_{1}, \mu_{2}, \mu_{3}, \mu_{4}\right)=(0,1,2,3),(0,1,2,2),(0,1,1,1)$ for $K=4$. We set up four types of arrangements of means $\left(\mu_{1}, \mu_{2}, \mu_{3}, \mu_{4}, \mu_{5}\right)=(0,1,2,3,4)$, $(0,1,2,3,3),(0,1,2,2,2),(0,1,1,1,1)$ for $K=5$. Specifically, for each $K$ we set up $K-1$ types of arrangements of means varying the number of differences among successive normal means from 1 to $K-1$. Although the formulations of the power of SS, SD and SU are obtained, we should obtain the power of CT and CT-W by Monte Carlo simulation. Here we obtain the power of CT and CT-W with 1,000,000 times of experiments. Tables 8 to 13 give the power of the test for five procedures. CT-W is most powerful among five procedures except for the case when the number of differences among successive normal means is equal to $K-1$. CT, SD and SU are more powerful compared to SS except for the case when the number of differences among successive normal means is equal to 1 . In each case the differences of power among $\mathrm{CT}, \mathrm{SD}$ and $\mathrm{SU}$ are not remarkably large.

Table 8 : Power of the test $(K=3, n=15)$

\begin{tabular}{c|ccccc}
\hline$\left(\mu_{1}, \mu_{2}, \mu_{3}\right)$ & SS & CT & CT-W & SD & SU \\
\hline$(0,1,2)$ & 0.546 & 0.704 & 0.710 & 0.706 & 0.711 \\
$(0,1,1)$ & 0.763 & 0.766 & 0.816 & 0.766 & 0.770 \\
\hline
\end{tabular}

Table 9 : Power of the test $(K=3, n=30)$

\begin{tabular}{c|ccccc}
\hline$\left(\mu_{1}, \mu_{2}, \mu_{3}\right)$ & SS & CT & CT-W & SD & SU \\
\hline$(0,1,2)$ & 0.938 & 0.972 & 0.972 & 0.972 & 0.972 \\
$(0,1,1)$ & 0.969 & 0.971 & 0.983 & 0.971 & 0.973 \\
\hline
\end{tabular}

Table 10 : Power of the test $(K=4, n=15)$

\begin{tabular}{c|ccccc}
\hline$\left(\mu_{1}, \mu_{2}, \mu_{3}, \mu_{4}\right)$ & SS & CT & CT-W & SD & SU \\
\hline$(0,1,2,3)$ & 0.290 & 0.579 & 0.588 & 0.578 & 0.596 \\
$(0,1,2,2)$ & 0.458 & 0.555 & 0.634 & 0.553 & 0.561 \\
$(0,1,1,1)$ & 0.712 & 0.713 & 0.743 & 0.714 & 0.714 \\
\hline
\end{tabular}

Table 11 : Power of the test $(K=4, n=30)$

\begin{tabular}{c|ccccc}
\hline$\left(\mu_{1}, \mu_{2}, \mu_{3}, \mu_{4}\right)$ & SS & CT & CT-W & SD & SU \\
\hline$(0,1,2,3)$ & 0.871 & 0.959 & 0.959 & 0.959 & 0.959 \\
$(0,1,2,2)$ & 0.913 & 0.942 & 0.957 & 0.942 & 0.944 \\
$(0,1,1,1)$ & 0.956 & 0.958 & 0.968 & 0.957 & 0.958 \\
\hline
\end{tabular}

Table 12 : Power of the test $(K=5, n=15)$

\begin{tabular}{c|ccccc}
\hline$\left(\mu_{1}, \mu_{2}, \mu_{3}, \mu_{4}, \mu_{5}\right)$ & SS & CT & CT-W & SD & SU \\
\hline$(0,1,2,3,4)$ & 0.133 & 0.468 & 0.481 & 0.467 & 0.501 \\
$(0,1,2,3,3)$ & 0.229 & 0.396 & 0.433 & 0.393 & 0.408 \\
$(0,1,2,2,2)$ & 0.399 & 0.485 & 0.509 & 0.463 & 0.466 \\
$(0,1,1,1,1)$ & 0.675 & 0.677 & 0.702 & 0.676 & 0.675 \\
\hline
\end{tabular}

Table 13 : Power of the test $(K=5, n=30)$

\begin{tabular}{c|ccccc}
\hline$\left(\mu_{1}, \mu_{2}, \mu_{3}, \mu_{4}, \mu_{5}\right)$ & SS & CT & CT-W & SD & SU \\
\hline$(0,1,2,3,4)$ & 0.791 & 0.945 & 0.946 & 0.945 & 0.946 \\
$(0,1,2,3,3)$ & 0.839 & 0.915 & 0.927 & 0.915 & 0.916 \\
$(0,1,2,2,2)$ & 0.891 & 0.923 & 0.933 & 0.915 & 0.916 \\
$(0,1,1,1,1)$ & 0.945 & 0.947 & 0.960 & 0.946 & 0.947 \\
\hline
\end{tabular}




\section{Conclusions}

In this study, we discussed the multiple comparison procedures for finding differences among a sequence of normal means with ordered restriction. Specifically, we proposed the stepwise procedure based on closed testing procedure using Williams (1971)'s statistics and compared our procedure with Lee and Spurrier (1995)'s single step procedure, Shiraishi (2014)'s stepwise procedure based on closed testing procedure, Douke et al. (2006)'s sequential rejective step down procedure and Imada (2015)'s step up procedure through the simulation regarding the power of the test. We confirmed that our procedure is most powerful in almost all cases. Specifically, by adopting the assumption that a sequence of normal means is ordered we can construct a powerful procedure.

We gave numerical examples when the number of the populations is not greater than 5. Although the determination of the power of closed testing procedure is accompanied with computational complications when the number of populations is large, we should give numerical examples regarding critical value and power of the test when the number of normal means is large in the future.

\section{Appendix}

In this Appendix, we derive

$$
P\left(t_{I_{1}}^{*} \leq c_{I_{1}, M}^{*}, t_{I_{2}}^{*} \leq c_{I_{2}, M}^{*}, \ldots, t_{I_{k}}^{*} \leq c_{I_{k}, M}^{*}\right) \geq \prod_{l=1}^{k} P\left(t_{I_{l}}^{*} \leq c_{I_{l}, M}^{*}\right)
$$

given in (7).

Let $U$ be a nonnegative random variable and $f(u)$ be its probability density function. Let $h_{1}(U), h_{2}(U), \ldots, h_{l}(U)$ be functions which are nonnegative, bounded, continuous and monotone in the same direction. Furthermore, we assume there exists finite expectation $E\left[h_{i}(U)\right]$ for each $i$. By Corollary A.1.1 in Hsu (1996)

$$
E\left[\prod_{i=1}^{l} h_{i}(U)\right] \geq \prod_{i=1}^{l} E\left[h_{i}(U)\right]
$$

(8) is rewritten as

$$
\int_{0}^{\infty} \prod_{i=1}^{l} h_{i}(u) f(u) d u \geq \prod_{i=1}^{l} \int_{0}^{\infty} h_{i}(u) f(u) d u
$$

We derive the inequality (7) using (9).

First, let $I=\{i, i+1, \ldots, j\}$. We consider the conditional probability $P\left(t_{I}^{*} \leq c_{I}^{*} \mid s_{0}\right)$ given $s_{0}$. Letting

$$
z_{0, l}=\frac{y_{l}-\bar{x}_{i}}{\sqrt{\frac{1}{n_{j}}+\frac{1}{n_{i}}} \sigma}(l=i+1, i+2, \ldots, j), \quad s_{0}=\frac{s}{\sigma},
$$

we obtain

$$
z_{l}=\frac{y_{l}-\bar{x}_{i}}{\sqrt{\frac{1}{n_{j}}+\frac{1}{n_{i}}} s}=\frac{z_{0, l}}{s_{0}}
$$


and

$$
P\left(t_{I}^{*} \leq c_{I}^{*} \mid s_{0}\right)=P\left(z_{0, i+1} \leq c_{I}^{*} s_{0}, z_{0, i+2} \leq c_{I}^{*} s_{0}, \ldots, z_{0, j} \leq c_{I}^{*} s_{0} \mid s_{0}\right) .
$$

Here $\left(z_{0, i+1}, z_{0, i+2}, \ldots, z_{0, j}\right)^{\prime}$ is distributed according to the multivariate normal $N_{j-i}\left(\mathbf{0}, \boldsymbol{\Lambda}_{I}\right)$. Letting $f_{0}\left(z_{0, i+1}, z_{0, i+2}, \ldots, z_{0, j} ; \boldsymbol{\Lambda}_{I}\right)$ be its probability density function, we obtain

$P\left(t_{I}^{*} \leq c_{I}^{*} \mid s_{0}\right)=\int_{-\infty}^{c_{I}^{*} s_{0}} \int_{-\infty}^{c_{I}^{*} s_{0}} \cdots \int_{-\infty}^{c_{I}^{*} s_{0}} f_{0}\left(z_{0, i+1}, z_{0, i+2}, \ldots, z_{0, j} ; \boldsymbol{\Lambda}_{I}\right) d z_{0, i+1} d z_{0, i+2} \cdots d z_{0, j}$

by (10). (11) shows that $P\left(t_{I}^{*} \leq c_{I}^{*} \mid s_{0}\right)$ is monotonically increasing for $0 \leq s_{0}<\infty$. By (9)

$$
\int_{0}^{\infty} \prod_{l=1}^{k} P\left(t_{I_{l}}^{*} \leq c_{I_{l}, M}^{*} \mid s_{0}\right) g\left(s_{0}\right) d s_{0} \geq \prod_{l=1}^{k} \int_{0}^{\infty} P\left(t_{I_{l}}^{*} \leq c_{I_{l}, M}^{*} \mid s_{0}\right) g\left(s_{0}\right) d s_{0} .
$$

Since

$$
\int_{0}^{\infty} P\left(t_{I_{l}}^{*} \leq c_{I_{l}, M}^{*} \mid s_{0}\right) g\left(s_{0}\right) d s_{0}=P\left(t_{I_{l}}^{*} \leq c_{I_{l}, M}^{*}\right)
$$

for $l=1,2, \ldots, k$, we obtain

$$
\int_{0}^{\infty} \prod_{l=1}^{k} P\left(t_{I_{l}}^{*} \leq c_{I_{l}, M}^{*} \mid s_{0}\right) g\left(s_{0}\right) d s_{0} \geq \prod_{l=1}^{k} P\left(t_{I_{l}}^{*} \leq c_{I_{l}, M}^{*}\right) .
$$

On the other hand, since $t_{I_{1}}^{*}, t_{I_{2}}^{*}, \ldots, t_{I_{k}}^{*}$ are independent when the value of $s_{0}$ is given, we obtain

$$
P\left(t_{I_{1}}^{*} \leq c_{I_{1}, M}^{*}, t_{I_{2}}^{*} \leq c_{I_{2}, M}^{*}, \ldots, t_{I_{k}}^{*} \leq c_{I_{k}, M}^{*}\right)=\int_{0}^{\infty} \prod_{l=1}^{k} P\left(t_{I_{l}}^{*} \leq c_{I_{l}, M}^{*} \mid s_{0}\right) g\left(s_{0}\right) d s_{0} .
$$

By (12) and (13) we obtain

$$
P\left(t_{I_{1}}^{*} \leq c_{I_{1}, M}^{*}, t_{I_{2}}^{*} \leq c_{I_{2}, M}^{*}, \ldots, t_{I_{k}}^{*} \leq c_{I_{k}, M}^{*}\right) \geq \prod_{l=1}^{k} P\left(t_{I_{l}}^{*} \leq c_{I_{l}, M}^{*}\right) .
$$

\section{Acknowledgement}

The author is deeply grateful to the referee and the editors for their valuable comments and suggestions.

\section{References}

Bartholomew, D. J. (1959). A test of homogeneity for ordered alternatives, Biometrika 46, 36-48.

Douke, H., Imada, T. and Nakamura, T. (2006). Development of sequential step-wise procedures for dose response test. Journal of the Japan Statistical Society 36 (1), 45-64. (in Japanese) 
Hayter, A. J. (1990). A one-sided studentized range test for testing against a simple ordered alternative, Journal of the American Statistical Association 85, No. 411, 778-785.

Hsu, J. C. (1996). Multiple Comparisons. Boca Raton : Chapman \& Hall.

Imada, T. (2015). Multiple comparison procedures for checking differences among sequence of normal means with ordered restriction. Journal of the Japan Statistical Society 44 (2), 251-270. (in Japanese)

Lee, R. E. and Spurrier, J. D. (1995). Successive comparisons between ordered treatments. Journal of Statistical Planning and Inference, 43, 323-330.

Nagata, Y. and Yoshida, M. (1997). Basic Theory of Statistical Multiple Comparison Procedures. Tokyo : Scientist Inc. (in Japanese)

Nashimoto, K. and Wright, F.T. (2005). Multiple comparison procedures for detecting differences in simply ordered means, Computational Statistics and Data Analysis, 48, 291-306.

Ramsey, P. H. (1978). Power differences between pairwise multiple comparisons. Journal of the American Statistical Association, 73, 479-485.

Shiraishi, T. (2014). Closed testing procedures in multi-sample models under a Simple Ordered Restriction. Journal of the Japan Statistical Society 43 (2), 215-245. (in Japanese)

Williams, D. A. (1971). A test for differences between treatment means when several dose levels are compared with a zero dose control, Biometrics 27, 103-117.

Received March 20, 2015

Revised September 4, 2015 